\title{
Prevalencia de huevos de Toxocara spp. en áreas recreacionales del distrito Gregorio Albarracín Lanchipa y el nivel de contaminación (ligero, moderado, alto)
}

\author{
Prevalence of Toxocara spp. eggs in recreational areas of Gregorio Albarracín \\ Lanchipa district and the level of pollution (light, moderate, high)
}

\author{
${ }^{1}$ Diana Farfán Pajuelo \\ ${ }^{2}$ Rosalía Quispe Quispe
}

\author{
${ }^{3}$ Anthony Rivera Prado \\ ${ }^{4}$ Luis Lloja Lozano
}

\section{RESUMEN}

El objetivo de la investigación consistió en determinar la prevalencia de huevos de Toxocara spp. en áreas recreacionales del distrito Gregorio Albarracín Lanchipa (Tacna-Perú) y el nivel de contaminación (ligero, moderado, alto). La población estuvo formada por 69 áreas recreativas (considerando parques, plazas y jardines), tomándose como muestra (al azar) a 10 de estas áreas. Para la recolección de muestras se utilizó el método de la "W". Se realizó el muestreo de suelo y/o césped, depositando cada muestra en una bolsa de polietileno. Posteriormente, se rotuló y transportó al Laboratorio de Parasitología de la Universidad Nacional Jorge Basadre Grohmann (UNJBG). Durante el procesamiento de la muestra, se empleó el método de flotación de Willis $\mathrm{NaCl}$ al $25 \%$ (P/P) y la observación microscópica para identificación, según las características morfológicas de la forma evolutiva parasitaria. Posteriormente, se realizó la categorización de acuerdo al grado de contaminación por número de huevos encontrados en la muestra. Los resultados se expresaron como número de huevos por cada $50 \mathrm{~g}$ de suelo. De acuerdo a los resultados, se determinó que, la prevalencia de Toxocara spp. es del $70 \%$. Con respecto a la contaminación del suelo, el $60 \%$ de áreas recreativas tienen un nivel de contaminación ligera, mientras que el $10 \%$ tienen un nivel de contaminación moderado y el $30 \%$ de áreas recreativas no presenta algún nivel de contaminación.

Palabras Claves: Infección parasitaria, Toxocara spp., zoonosis.

\section{ABSTRACT}

The objective of the research was to determine the prevalence of Toxocara spp. egges in recreational areas of the Gregorio Albarracín Lanchipa district (Tacna-Peru) and the pollution level: light, moderate, high. The population was formed by 69 recreational areas (considering parks, squares and gardens) from which 10 areas were taken as a random sample of 10 was taken. For the collection of samples, the "W" method was used.The soil and / or grass sampling was carried out by depositing it in a polyethylene bag. Later it was labeled and transported to the Parasitology Laboratory of the UNJBG. During the processing of the sample, the Willis $\mathrm{NaCl}$ flotation method at $25 \%$ ( $\mathrm{P} / \mathrm{P}$ ) was used, as well as the microscopic observation for identification according to the morphological characteristics of the parasitic evolutionary form. Subsequently, the categorization was done according to the degree of contamination by egg number found in the sample. The results were expressed as egg number per $50 \mathrm{~g}$ of soil. According to the results, it was determined that the prevalence of Toxocara spp. was of $70 \%$. Regarding soil contamination, $60 \%$ of recreational areas had a light level of pollution, while $10 \%$ had a moderate level of pollution and $30 \%$ of recreational areas did not show any level of contamination.

Keywords: Parasitic infection, Toxocara spp., zoonosis.

\footnotetext{
${ }^{1}$ Laboratorio de Parasitología. Facultad de Ciencias. Universidad Nacional Jorge Basadre Grohmann. Tacna-Perú. E-mail: dianafarfan23.df@gmail.com ${ }^{2}$ Laboratorio de Parasitología. Facultad de Ciencias. Universidad Nacional Jorge Basadre Grohmann. Tacna-Perú. E-mail: rosaliavq16@gmail.com ${ }^{3}$ Laboratorio de Parasitología. Facultad de Ciencias. Universidad Nacional Jorge Basadre Grohmann. Tacna-Perú. E-mail: brayandna64@gmail.com ${ }^{4}$ Laboratorio de Parasitología. Facultad de Ciencias. Universidad Nacional Jorge Basadre Grohmann. Tacna-Perú. E-mail: lullolo62@hotmail.com
} 


\section{INTRODUCCIÓN}

Los animales son considerados huéspedes de diversos parásitos de importancia en patogenia humana con alto potencial zoonótico, ya que estos albergan en su tracto intestinal gran cantidad de diversas especies de nematodos, cestodos y protozoos de carácter clínico. El término zoonosis hace referencia a las enfermedades e infecciones que se comparten entre animales vertebrados y humanos. En ese contexto, los lugares de esparcimiento que se comparten entre animales y el hombre, caso de los parques públicos, constituyen un punto de contaminación zoonótica por la mala higiene e inadecuado control de heces y excretas caninas.

Por lo cual es muy importante, realizar estudios de contaminación parasitaria del suelo, ya que este constituye un indicador directo del riesgo de infección al que están expuestas las personas que visitan frecuentemente los parques (Córdoba, Ciarmela, Pezzani, Gamboa, De Luca, Minvielle \& Basualdo, 2002).

\section{METODOLOGÍA}

Con respecto al área de estudio, el distrito de Coronel Gregorio Albarracín Lanchipa se encuentra ubicado en el llamado "Cono Sur" de la provincia de Tacna, cuya capital es la Villa Alfonso Ugarte. Su jurisdicción está configurada sobre la demarcación del entonces Centro Poblado Menor Nueva Tacna. Su extensión es de $187.74 \mathrm{~km}^{2}$, representando aproximadamente el $1.2 \%$ de la extensión departamental y el $2.4 \%$ de la extensión total de la provincia de Tacna. Con respecto a su altitud, se encuentra ubicado aproximadamente a $560 \mathrm{msnm}$ (Municipalidad distrital Gregorio Albarracín Lanchipa, 2009)

Norte: Inicia en la intersección de la avenida la Bohemia Tacneña con la avenida Collpa, en el Ovalo Tarapacá.

Sureste: El límite se inicia en el cauce de la quebrada de Cauñani, continua en dirección Sur Oeste, agua abajo por el cauce de la quebrada de Cauñani.

Este: El límite se inicia en la intersección de la avenida Gregorio Albarracín con la avenida Collpa.

Oeste: El límite se inicia en la intersección de la quebrada de Cauñani con la carretera Panamericana.

\section{Unidad de análisis}

La unidad de análisis es la muestra de cada punto de muestreo del suelo de parques, jardines y plazas públicas del distrito de Gregorio Albarracín Lanchipa.

\section{Población y muestra}

La población está formada por 69 áreas recreativas (considerando parques, plazas y jardines), de los cuales se tomó como muestra al azar a 10 de ellas (Tabla 1). Con un muestreo no probabilístico intencional o de conveniencia. Para realizar una mejor distribución de los 10 parques a elegir, el área de estudio fue dividido en estratos, debido a que la población era heterogénea con respecto al tamaño del parque, jardín o plaza (Variables: nivel de contaminación de cada parque y la prevalencia de parásitos en cada parque muestreado).

\section{Tamaño de las áreas recreativas}

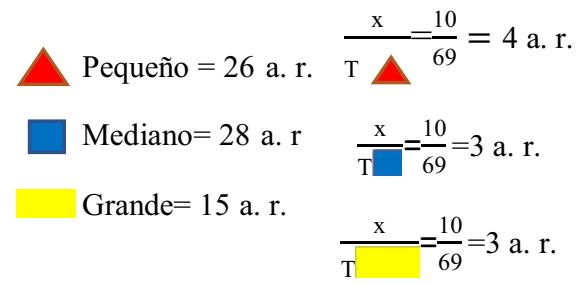

10 muestra ----69 población

*Donde a.r.: áreas recreativas

Tabla 1. Relación de las áreas recreativas consideradas para estudio en el distrito Gregorio Albarracín Lanchipa, Tacna

\begin{tabular}{|cl|}
\hline Numero & \multicolumn{1}{c|}{ Nombre } \\
\hline $\mathbf{1}$ & Plaza Jorge Chávez \\
$\mathbf{2}$ & Plaza Federico Ríos \\
$\mathbf{3}$ & PlazaLas Américas \\
$\mathbf{4}$ & Plaza Santa Rosa de Lima \\
$\mathbf{5}$ & PlazaLas Violetas \\
$\mathbf{6}$ & Plaza Margaritas \\
$\mathbf{7}$ & Plaza Pérez Gamboa \\
$\mathbf{8}$ & ParqueLas Aralias \\
$\mathbf{9}$ & Parque Amapolas \\
$\mathbf{1 0}$ & ParqueLos Laureles \\
\hline
\end{tabular}

\section{MATERIAL Y MÉTODOS}

\section{Material biológico}

- Muestra de tierra y/o césped

\section{Material de laboratorio \\ Reactivos}

- Solución de $\mathrm{NaCl}$ al 25 \% (P/P) 
- Lugol parasitológico

- Agua destilada

Material y equipo

- Centrifuga

- Microscopio

- Balanza analítica

- Guantes desechables

- Mascarillas desechables

- Envases de plástico de $250 \mathrm{ml}$ boca ancha

- Bagueta

- Colador

- Vasos de precipitación

- Probeta

- Tubos de ensayo

- Gasa

- Goteros Pasteur

- Gradilla

- Laminas portaobjetos y cubreobjetos

\section{Materiales de campo:}

-Libreta

- Pala pequeña

- Bolsas de polietileno

- Bolsas de almacenamiento

- Tijera

- Cinta Masking Tape

\section{Tipo y diseño de la investigación}

El estudio es de tipo descriptivo, transversal (debido a que la observación fue en un momento dado) y prospectivo. El diseño de la investigación es diseño no experimental porque no se manipularon las variables (Morales, 2014).

\section{Método de la investigación}

Para el muestreo, se usó el método de la doble "W" en parques y jardines (Morales, 2014). Se determinó la longitud de cada "W" en pasos y estimando en forma proporcional el número de puntos de muestreo (dependiendo del tamaño de cada cuadrante). (Cáceres, Bustinza \& Valderrama, 2017). Para procesar las muestras se empleó el método de flotación de Willis $\mathrm{NaCl}$ al $25 \%(\mathrm{P} / \mathrm{P})$

\section{Diseño procedimental}

\section{Obtención de la muestra del suelo}

En cada punto de muestro se recogió 500 gramos con ayuda de una pequeña pala, para formar una muestra de $6 \mathrm{~kg}$ a $8 \mathrm{~kg}$ en cada recorrido, considerando que cada parque se subclasificó en cuadrantes. La muestra fue de un área de $135 \mathrm{~cm}^{2}$ y a una profundidad de $5 \mathrm{~cm}$ a $7 \mathrm{~cm}$. Se determina la cantidad de puntos de muestreo según el tamaño de cada cuadrante.

Las muestras de suelo y/o césped se colocaron en bolsas de polietileno rotuladas y conservadas a temperatura ambiente durante 2 a 3 días hasta su análisis parasitológico (Morales, 2014).

\section{Procedimiento de campo}

Se realizó el muestreo de suelo y/o césped depositándola en una bolsa de polietileno. Posteriormente, se rotuló y transportó al Laboratorio de Parasitología de la UNJBG para su respectivo análisis.

\section{Análisis parasitológico}

El análisis parasitológico de las muestras de suelo, luego de la técnica empleada, método de Wilis al $25 \%(\mathrm{P} / \mathrm{P})$, fue realizada por observación microscópica. Donde se identificó, de acuerdo a las características que presentaba, la forma evolutiva del parásito encontrado y para el grado de contaminación del suelo se clasificó de acuerdo al número de huevos observados en la muestra en ligera (de 1 a 5 huevos), moderada (de 6 a 10 huevos) e intensa (más de 10 huevos) en las láminas montadas por muestra (4 láminas por muestra.) Los resultados se expresaron como número de huevos/50 g de suelo. (Morales, 2014)

\section{Procedimiento de laboratorio: Laboratorio de Parasitología de UNJBG)}

\section{Método de flotación con $\mathrm{NaCl} 25 \%(\mathrm{P} / \mathrm{P})$ : (Método de Willis)}

La técnica de flotación con $\mathrm{NaCl}$ al $25 \%(\mathrm{P} / \mathrm{P})$, técnica de flotación y sedimentación para determinación de huevos y estados larvarios de helmintos (Cáceres et al., 2017).

1. Se homogenizó la muestra por punto con ayuda de una tijera. Luego se juntó las muestras según el cuadrante perteneciente.

2. Se tomó $50 \mathrm{~g}$ de dicha muestra de cada cuadrante de parque, jardín o plaza y se añadió 50 $\mathrm{ml}$ de agua corriente. Se homogenizó.

3. Se filtró usando un colador y un tamiz triple de gasa. 
4. La muestra filtrada se repartió a un tubo de ensayo de $10 \mathrm{ml}$ de capacidad. Se centrifugó a 2 400 rpm por 4 minutos.

5. Posteriormente, se eliminó el sobrenadante y se volvió a repetir el lavado 3 veces.

6. Se los depósitos en viales de vidrio de $60 \mathrm{ml}$.

7. Luego se volvió a centrifugar, se eliminó el sobrenadante y se le añadió la solución de $\mathrm{NaCl}$ al $25 \%(\mathrm{P} / \mathrm{P})$. Se centrifugó.

8. Con la ayuda de un gotero se adicionó el $\mathrm{NaCl}$ al $33 \%$, con el fin de formar un menisco convexo sobre el cual se colocó una lámina cubreobjetos.

9. Luego de 15 minutos se colocó la lámina cubreobjetos a una lámina portaobjetos. Tinción con 1 gota Lugol.

10. Seguidamente, se hizo la lectura al microscopio en objetivos 10x y 40x.

11. Finalmente, se interpretó según grado de contaminación expresado como número de huevos/50 gramos de suelo.

\section{Para determinar la prevalencia}

Se tabuló los datos de la positividad obtenida en cada parque, mediante el programa Excel. Luego, se procedió a calcular la prevalencia de parásitos zoonóticos de los parques y plazas públicas del distrito de Gregorio Albarracín Lanchipa (Miranda, 2017).

$$
\text { Prevalencia }=\frac{\mathrm{N}^{\circ} \text { de parques positivos }}{\mathrm{N}^{\circ} \text { total de parques }} \times 100
$$

\section{RESULTADOS}

Contaminación en áreas recreativas (plazas, parques y jardines públicos) con huevos de Toxocara spp. en el distrito de Gregorio Albarracín Lanchipa.

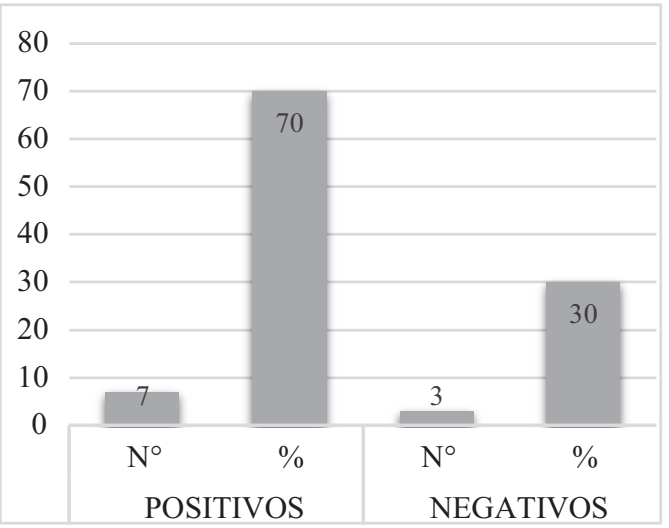

Figura 1. Prevalencia, huevos de Toxocara sp. en plazas, parques y jardines públicos del distrito de Gregorio Albarracín Lanchipa, Tacna 2018.
En la Figura 1 se observó que la prevalencia positiva a huevos de Toxocara spp. fue del $70 \%$ en todas las plazas, parques y jardines estudiados en el distrito Gregorio Albarracín Lanchipa. Esto se debió a que el análisis se hizo por cada cuadrante de cada uno de las áreas recreativas (un total de 93 láminas a observar), obteniendo una muestra más diversa. Asimimismo, dio como prevalencia negativa un $30 \%$, esto debido a que estos son parásitos de mayor distribución en diferentes especies de animales, en especial perros.

Tabla 2. Nivel de contaminación por Toxocara spp. encontrados en plazas, parques y jardines públicos del distrito de Gregorio Albarracín Lanchipa, Tacna 2018

\begin{tabular}{ccc}
\hline & $\mathbf{N}^{\circ}$ & \% \\
\hline Plaza 1 & 10 & 43.48 \\
\hline Plaza 2 & 1 & 4.35 \\
\hline Plaza 3 & 5 & 21.74 \\
\hline Plaza 4 & 1 & 4.35 \\
\hline Plaza 5 & 0 & 0.00 \\
\hline Plaza 6 & 1 & 4.35 \\
\hline Plaza 7 & 1 & 4.35 \\
\hline Plaza 8 & 0 & 0.00 \\
\hline Plaza 9 & 0 & 0.00 \\
\hline Plaza 10 & 4 & 17.39 \\
\hline
\end{tabular}

Donde:
Contaminación ligera
Contaminación moderada
Contaminación intensa

NIVEL DE CONTAMINACION DEL SUELO

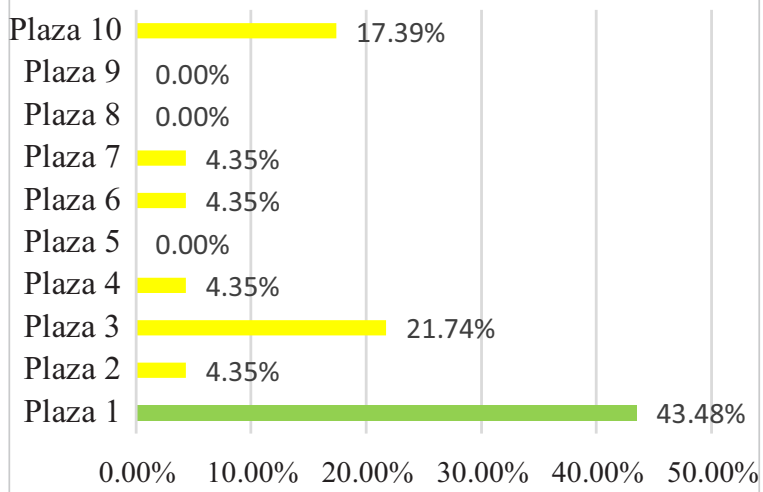

Figura 2. Nivel de contaminación por Toxocara spp. encontrados en plazas, parques y jardines públicos del distrito de Gregorio Albarracín Lanchipa, Tacna 2018. 1)Plaza Jorge Chávez, 2) Plaza Federico Ríos, 3) Plaza Las Américas, 4) Plaza Santa Rosa de Lima, 5) Plaza Las Violetas, 6) Plaza Margaritas, 7) Plaza Pérez 
Gamboa, 8) Parque Las Aralias, 9) Parque Amapolas, 10) Parque Los Laureles.

Se observó que, la Plaza Jorge Chávez tiene un nivel de contaminación moderada de parásitos con 43.48 \%; mientras que el Parque Los Laureles tiene un nivel de contaminación moderada con $17.39 \%$, Plaza Las Américas presenta un nivel de contaminación moderada con $21.74 \%$. Por otro lado, la Plaza Pérez Gamboa, Plaza Margaritas, Plaza Santa Rosa de Lima y Plaza Federico Ríos tienen un nivel de contaminación bajo con $4.35 \%$. Por lo contrario, se muestra que el Parque Amapolas, el Parque las Aralias y la Plaza las Violetas no mostraron presencia de Toxocara spp.

Tabla 3. Nivel de contaminación de las plazas, parques y jardines públicos del distrito de Gregorio Albarracín Lanchipa, Tacna 2018

\begin{tabular}{|ccc|}
\hline Nivel & Frecuencia & \% \\
\hline Ligera & 6 & 60 \\
Moderada & 1 & 10 \\
Intensa & 0 & 0 \\
TOTAL & 10 & \\
\hline
\end{tabular}

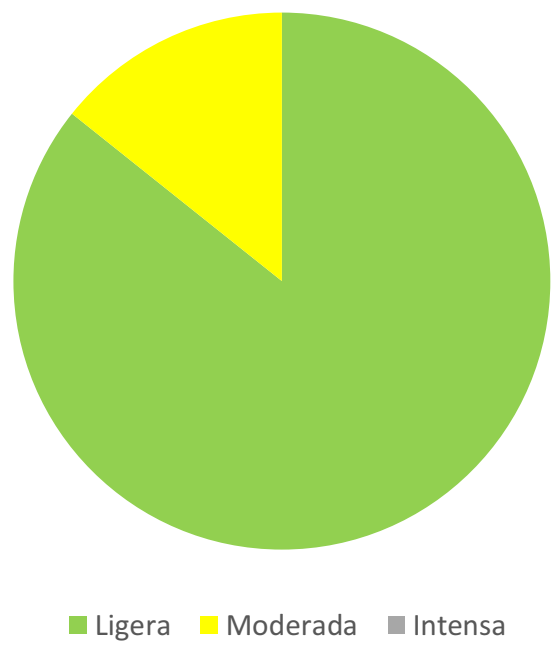

Figura 3. Nivel de contaminación de las plazas, parques y jardines públicos del distrito de Gregorio Albarracín Lanchipa, Tacna 2018.

Se puede observar que el $60 \%$ de áreas recreativas tienen un nivel de contaminación ligero, mientras que el $10 \%$ tienen un nivel de contaminación moderado y el $30 \%$ de áreas recreativas no presenta algún nivel de contaminación.

\section{Parásitos identificados en las áreas recreativas}

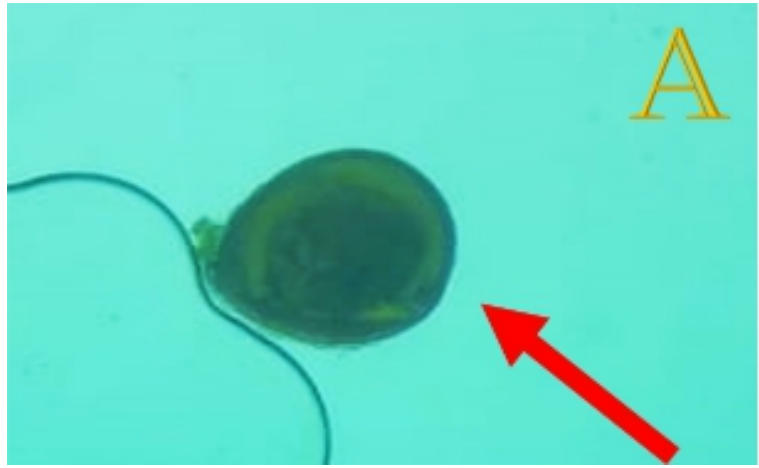

Figura 4. Huevo no embrionado de Toxocara sspp.

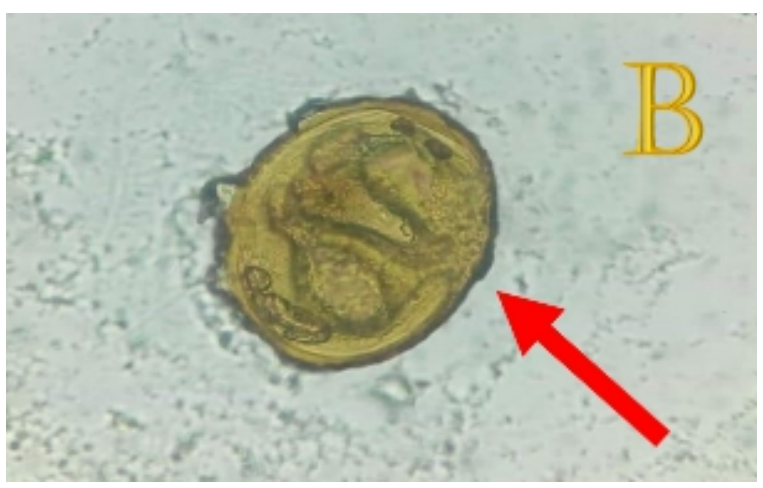

Figura 5. Huevo larvado de Toxocara sspp.

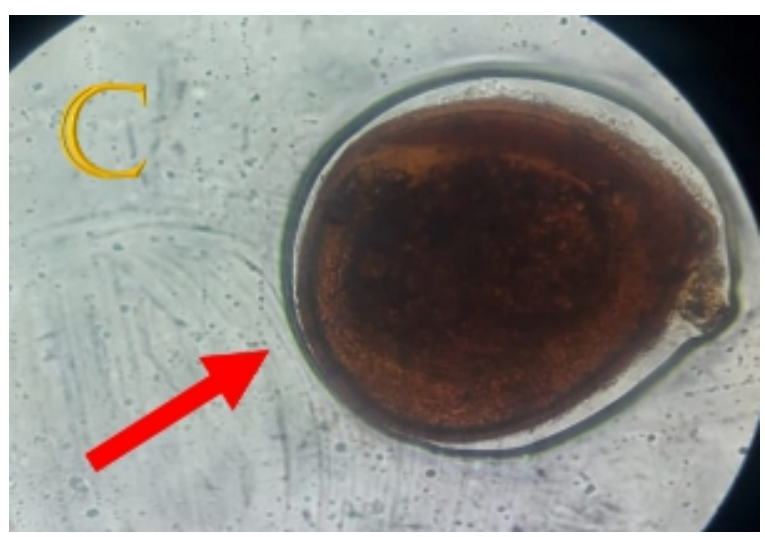

Figura 6. Huevo no embrionado de Toxocara sspp.

\section{DISCUSIÓN}

El presente trabajo obtiene los fundamentos para su desarrollo en la importancia clínicoepidemiológica de los lugares de esparcimiento 
público de nuestra ciudad, al considerarse uno de los principales focos de infección de parásitos implicados en patogenia humana procedentes principalmente de excretas de animales domésticos. El desconocimiento de esta situación limita las acciones tomadas por las instituciones responsables del manejo y administración de los parques públicos.

La transmisión de zoonosis parasitarias a humanos se puede producir de forma directa por heces diseminadas o puede estar asociada con factores socioculturales; como la falta de hábitos higiénicos, carencia de instalaciones adecuadas, falta de control en el manejo de mascotas y animales callejeros (Iannacone, Alvariño, \& Cárdenas, 2012). La contaminación de espacios públicos debido a heces fecales facilita la transmisión (Marder, Ulon, Bottinelli, Meza, Lotero \& Ruiz, 2004) y compromete seriamente la salud humana (Iannacone et al., 2012).

Los lugares que tienen mayor contaminación parasitaria son las áreas recreativas (parques, plazas y jardines). Estos son visitados frecuentemente por personas con sus mascotas y constituyen un sitio de recreación para los habitantes de las ciudades. Los niños son considerados la población más susceptible por llevar a cabo, entre otras acciones, prácticas de geofagia (ingesta de tierra) (Iannacone et al., 2012).

Mediante esta investigación se busca mostrar la situación actual de las áreas recreativas analizadas del distrito Gregorio Albarracín Lanchipa, con la finalidad de crear una línea de trabajo base para la posterior búsqueda de soluciones y medidas de prevención en salud, teniendo en cuenta que estos parásitos son transmitidos por animales domésticos (mascotas). Por ello, el control y la desparasitación de estos animales, ya sean mascotas o en condición de abandono, sería la medida de acción más importante a considerar para la posteridad.

Se eligió el método de muestreo en W, ya que es considerado el más representativo para establecer la contaminación por huevos de parásito en una superficie de tierra (Morales, 2014). A su vez, se clasificaron los parques en estratos diferenciándolos en tamaños, ya que se reporta que los parques públicos con mayor área presentan mayores niveles de contaminación (Iannacone et al., 2012). Se utiliza el método de Willis, ya que es utilizado para separar los parásitos en todos sus estadios de otros objetos, basados en sus diferentes densidades. (Cáceres, 2017)
Los huevos de helmintos de importancia en salud pública encontrados fueron del género Toxocara spp., con una prevalencia del $70 \%$ en plazas, parques y jardines públicos del distrito de Gregorio Albarracín Lanchipa. Lo mencionado se debe a que son parásitos de mayor distribución en diferentes especies de animales, especialmente en perros. Con respecto a los resultados de otros estudios similares sobre la prevalencia de huevos de Toxocara spp., se obtuvieron datos similares. Por ejemplo, en Lima, los resultados obtenidos en laboratorio mostraron que existe prevalencia positiva de $28.6 \%$ de Toxocara spp. en parques (Carrasco, 2014). En Cusco, se concluyó que la prevalencia de Toxocara canis es de $61.29 \%$ (Bravo, 2015). En Trujillo, resultados obtenidos muestran una prevalencia positiva de $28 \%$ a huevos de Toxocara canis en parques, que determinaría un nivel de contaminación moderado (Requena, 2015). En la ciudad de Juliaca, investigaciones muestran que la prevalencia general de Toxocara spp en parques fue de $51.67 \%$ (Quenaya, 2017). En Ayacucho, se comprobó que $71 \%$ de áreas recreacionales se encuentran contaminadas con huevos de Toxocara spp. (Oras. 2012). En Tacna, la prevalencia de Toxocara spp en parques públicos fue de $53.8 \%$ (Alania, 2004). De los resultados obtenidos podemos decir que la prevalencia de Toxocara spp. es elevada, encontrándose por encima del promedio nacional que es de $52.5 \%$ (Iannacone et al., 2012), asimismo, la condición de parques negativos a Toxocara no indica que esta sea su condición real, sino que la cantidad relativa de huevos es menor al límite inferior de detección de los métodos utilizados (Canese, Domínguez, Otto, Ocampos \& Mendonca, 2003)

Esta prevalencia se puede atribuir a que los huevos de Toxocara canis presentan una cubierta gruesa, la que proporciona una gran resistencia a las condiciones adversas, como la presencia de productos químicos y la falta de humedad, por lo que pueden permanecer viables hasta 5 años. Esto le confiere una mayor capacidad de diseminación en el suelo y por lo tanto un mayor riesgo potencial de infección independiente de la época del año, por lo que generalmente su frecuencia no tiene una marcada estacionalidad, como ha sido demostrado en varios estudios (Peña, Vidal, del Toro, Hernández, Zapata, 2017).

Los huevos están expuestos a la desecación y a los rayos solares que los destruyen en corto tiempo y a la presencia de canes que deambulan en los lugares de estudio, esto explicaría nuestros 
resultados. Este elevado nivel de contaminación del suelo de las áreas recreacionales evaluadas con heces de perros con Toxocara spp. confirma los resultados de estudios previos, que señalan al suelo como fuente de infección de Toxocariosis en seres humanos, en especial en niños por sus hábitos de juego y sus inadecuadas medidas de higiene (Guardis, Radman y Archelli, 2000).

Estas variaciones en cuanto a la prevalencia de los parásitos en los distintos lugares de estudio, incluso entre regiones de una misma área geográfica, se debería a diversos factores, entre ellos destaca el aumento del número de mascotas. Según Ríos (2002), quien señala la existencia de 01 perro por cada 10 personas (considerándose los reportes del INEI), mostrándose un crecimiento exponencial en estos últimos años, sobre todo en lugares de recreación como son las plazas, parques y jardines. Además, otro factor sería el estado en el que se encuentran esos espacios públicos.

Respecto a los niveles de contaminación, cabe mencionar que este parámetro se encuentra influenciado directamente por factores indistintos a la limpieza de las áreas recreacionales, condiciones de higiene o medidas sanitarias. Por otro lado el mantenimiento de áreas recreacionales es otro factor importante en el desarrollo y presencia de huevos de parásitos, se confirma lo mencionado por Liñán y Castellanos (2010).

\section{CONCLUSIONES}

La prevalencia de Toxocara spp., que se presentan en cada parque muestreado del distrito Gregorio Albarracín Lanchipa, es del $70 \%$.

En el nivel de contaminación del suelo, se muestra que el $60 \%$ de las áreas recreacionales muestreadas tienen un nivel de contaminación ligera, el $10 \%$ tiene un nivel de contaminación moderada y el $30 \%$ restante no presenta.

\section{REFERENCIAS BIBLIOGRÁFICAS}

Alania, A. (2004). Contaminación de parques públicos con huevos de Toxocara spp. en la zona urbana de Tacna (tesis de pregrado). Universidad Nacional Jorge Basadre Grohmann, Tacna.
Bravo, W. (2015). Contaminación de los suelos en los parques del distrito de Wanchaq, Cusco. Con Toxocara Canis, Cusco 2015 (tesis de pregrado). Universidad Católica de Santa María, Arequipa.

Cáceres, C., Bustinza, R. \& Valderrama, A. (2017). Contaminación con Huevos de Toxocara sp y Evaluación Sanitaria de Parques en la Ciudad de Abancay, Perú. Revista de Investigaciones Veterinarias del Perú, 28(2), 376-386. doi:10.15381/rivep.v28.i2.13064.

Carrasco, V. (2015). Determinación de la presencia de 7 huevos de Toxocara sp. $Y$ Ancylostoma sp. en parques del distrito de Barranco-Lima - Perú 2014 (tesis de pregrado). Universidad Científica del Sur, Lima.

Canese, A., Domínguez, R., Otto, C., Ocampos, C. \& Mendonca, E. (2003). Huevos infectivos de toxocara, en arenas de plazas y parques de Asunción, Paraguay. Revista chilena de pediatria, 74(6), 611-616. d o i : $10.4067 / \mathrm{S} 0370$ 41062003000600010

Córdoba, A., Ciarmela, M., Pezzani, B., Gamboa, M., De Luca, M., Minvielle, M., \& Basualdo, J. (2002). Presencia de parásitos intestinales en paseos públicos urbanos en La Plata Argentina. Parasitología latinoamericana, 57(1-2), 25-29. d o i : $10.4067 / \mathrm{S} 0717$ 77122002000100007

Fonrouge, R., Guardis, M., Radman, N. y Archelli, S. (2000), Contaminación de suelos con huevos de Toxocara spp. en plazas y parques públicos de la ciudad de La Plata, Buenos Aires, Argentina, Boletín chileno de parasitología, 55(34), 83-85. doi:10.4067/S036594022000000300009

Iannacone, L., Alvariño, L. \& Cárdenas, J. (2012). Contaminación de los suelos con huevos de toxocara canis en parques públicos de Santiago de Surco, Lima, Perú 2007-2008. Neotropical Helminthology, 6(1), 97-108. Recuperado de https://dialnet.unirioj a.es/servlet/articulo? codigo $=3974849$

Liñan, R. y Castellanos, R. (2010). Contaminación con huevos de Toxocara canis en plazas y parques públicos del distrito Gregorio 
Albarracín Lanchipa. REBIOL, 30(1), $65-68$. R e c u p e r a d o d e http://www.facbio.unitru.edu.pe/inde x.php?option $=$ com_content $\&$ view $=$ ar ticle\&id $=66 \&$ Itemid $=98$

Marder, G., Ulon, S., Bottinelli, O., Meza, Z., Lotero, D., \& Ruiz, R. (2004). Infestación parasitaria en suelos y materia fecal de perros y gatos de la ciudad de Corrientes. Revista Veterinaria, 15(2), 70-72. Recuperado de http://revistas.unne.edu.ar/index.p $\mathrm{hp} / \mathrm{vet} /$ article/view/1999

Miranda, T. (2017). Contaminación por parásitos de importancia zoonótica en parques y plazas públicas del distrito de Miraflores, Arequipa-2017 (tesis de pregrado). Universidad Nacional de San Agustín de Arequipa.

Morales, M. (2014). Contaminación del suelo de viviendas y parques con huevos de entero parásitos de importancia en salud pública en el distrito de Alta de la Alianza- Tacna 2013 (tesis de pregrado). Universidad Nacional Jorge Basadre Grohmann, Tacna, Perú.

Municipalidad distrital Gregorio Albarracín Lanchipa. (2009). Plan de desarrollo concertado distrito Crnl. Gregorio Albarracín Lanchipa 2009 - 2016 [Ebook] (pp. 21-24). Tacna. Recuperado de http://www.peru.gob. pe/docs/PLANES/12667/PLAN_126 67 Plan de Desarrollo Concertado --_MDCGĀL__2009_2011.pdf.
Oras, Y. (2012). Presencia de huevos de Toxocara spp. en parques públicos durante los meses de agosto y setiembre (tesis de pregrado). La Universidad Nacional de San Cristóbal de Huamanga, Ayacucho.

Peña, I., Vidal, F., del Toro, A., Hernández, A. y Zapata, M. (2017) Zoonosis parasitarias causadas por perros y gatos, aspecto a considerar en Salud Pública de Cuba. Revista Electrónica de Veterinaria, 18(10), 1-11. Recuperado de http://www.redalyc.o rg/articulo.oa?id=63653470002

Quenaya, V. (2017). Toxocariosis canina y contaminación de parques de la ciudad de Juliaca con huevos de Toxocara spp. (tesis de pregrado). Universidad Nacional del Altiplano, Puno.

Requena, N. (2015). Nivel de contaminación de los parques recreacionales con huevos de Toxocara spp en el distrito La Esperanza, Trujillo, Perú. Eneromarzo 2015 (tesis de pregrado). Universidad Privada Antenor Orrego, Trujillo, Perú.

Ríos, A. (2002). Parasitismo gastrointestinal en caninos (Canis familiaris) en Tacna. (Tesis de pregrado). Universidad Nacional Jorge Basadre de Grohmann, Tacna, Perú. 